\title{
Características hematológicas de teleósteos brasileiros. II. Parâmetros sangüíneos do Piaractus mesopotamicus Holmberg (Osteichthyes, Characidae) em policultivo intensivo
}

\author{
Marcos Tavares-Dias ${ }^{1,2}$ \\ Rosilene A. Tenani ${ }^{1}$ \\ Leonardo D. Gioli ${ }^{1}$ \\ Christiane D. Faustino ${ }^{1}$
}

\begin{abstract}
Hematological characteristics of Brazilian teleosts. II. Blood parameters of Piaractus mesopotamicus Holmberg (Osteichthyes, Characidae) in an intensive polyculture system. Hematological parameters of freshwater fish Piaractus mesopotamicus (Holmberg, 1887) kept in an intensive polyculture system were determined. The correlations among these parameters as well as those between the parameters and the biometric data (total weight and standard length) were also analyzed. Erythrocytes count was $1.708 \pm 0.42 \times 10^{6} / \mu \mathrm{l}$, hemoglobin $6.6 \pm 2.0 \mathrm{~g} \%$ of blood and hematocrit $32.5 \pm 4.6 \%$, mean corpuscular volume (MCV) $209.8 \pm 69.5 \%$, mean corpuscular hemoglobin $(\mathrm{MCH}) 40.2 \pm 5.6 \mathrm{pg}$ and mean corpuscular hemoglobin concentration (MCHC) $21.4 \pm 8.4 \%$. The percentage of defense blood cells including leucocytes and thrombocytes, was studied. The percentage of thrombocytes was $61.4 \pm 10.0 \%$, lymphocytes $21.7 \pm 1.8 \%$, neutrophils $10.4 \pm 11.0 \%$, monocytes $4.7 \pm 3.3 \%$ and special granulocitic cells $1.8 \pm 2.0 \%$. The number of erythrocytes was positively correlated with the hemoglobin rate and negatively correlated with MVC. Hemoglobin was positively correlated with $\mathrm{MCHC}$ whereas hematocrit was negatively correlated with MCHC and MVC. Contrarily, leukocytes did not show correlations among themselves or with the hematological parameters and biometric data.

KEY WORDS. Piaractus mesopolamicus, blood, erythrocytes, freshwater fish, leucocytes, thrombocytes
\end{abstract}

O pacu, Piaractus mesopotamicus (Holmberg, 1887) é uma espécie da família Characidae, subfamília Myleinae de maior potencial para a aqüicultura brasileira, uma vez que apresenta grande habilidade de ganho de peso e adaptabilidade aos sistemas aqüaculturais (CASTAGNOLLI 1992). Apesar de sua importância econômica, nada tem sido feito em relação ao estudo de parâmetros hematológicos dessa espécie nas diferentes formas de cultivo empregado em pisciculturas brasileiras.

Zuim et al. (1988) estudaram a influência do meio ambiente sobre o percentual do hematócrito em P. mesopotamicus. Martins et al. (1995) utilizando aquários de 100 litros d'água investigaram os parâmetros sangüíneos em pacus alimentados com diferentes níveis de ácido ascórbico.

1) Departamento de Ciências Biológicas, Universidade de Franca. Avenida Dr. Armando Salles Oliveira 201, 14404-600 Franca, São Paulo, Brasil.

2) Laboratório de Patologia de Organismos Aquáticos, Centro de Aqüicultura, Universidade Estadual Paulista. Rodovia Carlos Tonanni Km 05, 14870-000 Jaboticabal, São Paulo, Brasil. 
Parâmetros hematológicos de peixes podem ser de grande valia em pisciculturas, uma vez que podem ser utilizados como indicadores do seu estado fisiológico, assim como no controle de patologias e estresse de manipulação (ALDRIN et al. 1982). Por essa razão, o presente trabalho tem como objetivo descrever os parâmetros hematológicos em Piaractus mesopotamicus, criados em sistema de policultivo intensivo.

\section{MATERIAL E MÉTODOS}

Neste trabalho 15 exemplares de Piaractus mesopotamicus Holmberg, 1887 foram capturados de um viveiro de $600 \mathrm{~m}^{2}$ da fazenda São Francisco $\left(20^{\circ} 26\right.$ 'S, $47^{\circ} 19^{\prime} \mathrm{W}$ ), Franca, São Paulo (Brasil), em abril de 1997. Os animais mantidos em sistema de policultivo intensivo foram alimentados com ração extrusada contendo $28 \%$ de proteina bruta.

Os animais, identificados como juvenis, tiveram $0,5 \mathrm{ml}$ de sangue coletado através de punção caudal com auxílio de seringas contendo EDTA $10 \%$. Em seguida, de cada exemplar anotou-se o peso total e comprimento padrão e os peixes foram então sacrificados por comoção cerebral através de incisão na linha média ventral com exposição das vísceras, para estudo microscópico da presença de parasitas. A presença ou não de ecto e endoparasitas foi verificada a fresco, utilizando-se muco da superfície do corpo e fragmentos de brânquias, rim, fígado, baço e coração, entre lâmina e lamínula. Também examinou-se a cavidade gastrointestinal, com auxílio de microscópio esteroscópico.

As primeiras gotas de sangue foram utilizadas na confecção de extensões sangüíneas em lâminas, as quais foram coradas segundo método de ROSENFELD (1947) e empregadas na contagem diferencial de células sangüíneas de defesa orgânica (incluindo leucócitos e trombócitos).

A contagem total de eritrócitos foi efetuada em câmara de Neubauer após diluição do sangue em solução contendo $\mathrm{NaCl}(0,65 \%)$ e vermelho neutro $(1 \%)$. Para estimar o volume de sangue ocupado pelas células em relação ao sangue total foi empregado o método de GoLDENFARB et al. (1971). A determinação da taxa de hemoglobina foi realizada através do método de Collier (1944). O volume corpuscular médio (VCM), hemoglobina corpuscular média (HCM) e a concentração de hemoglobina corpuscular média (CHCM) foram calculados segundo método preconizado por WINTROBE (1934).

Os resultados da correlação linear de Pearson foram analisados pelo teste $\mathrm{F}$, ao nível de 5\% de probabilidade, segundo BANZATO \& KRONKA (1995).

\section{RESULTADOS}

De acordo como as análises microscópicas de órgãos e superfície corporal, nenhum dos espécimes utilizados apresentou endo ou ectoparasitas ou lesões externas. A temperatura da água no momento da coletada sangüínea foi igual a $19^{\circ} \mathrm{C}$ e o $\mathrm{pH} 8,3$.

A amplitude de variação, média e intervalo de confiança dos parâmetros biológicos e da contagem total dos eritrócitos, taxa de concentração da hemoglobina, percentual de hematócrito, VCM, HCM e CHCM de P. mesopotamicus em policul- 
tivo intensivo estão relacionados na tabela I. Verifica-se que o número de eritrócitos variou de 0,950 a $2,290 \times 10^{6} / \mu \mathrm{l}$, a hemoglobina de 4,9 a $12,1 \mathrm{~g} \%$ de sangue, hematócrito de 24,0 a $45,0 \%$, VCM de 130,0 a $355,5 \%$, HCM de 27,2 a 55,0 pg e o CHCM 11,3 a 40,3\%.

Tabela I. Amplitude de variação (Ax), média \pm desvio padrão (SD) e intervalo de confiança (IC) dos parâmetros eritrocitários em $P$. mesopotamicus, mantido em policutivo intensivo.

\begin{tabular}{lrrr}
\hline \multicolumn{1}{c}{ Parâmetros $(\%)$} & Ax & Média \pm SD & \multicolumn{1}{c}{ IC } \\
\hline Peso total $(\mathrm{g})$ & $70,000-129,00$ & $91,00 \pm 34,40$ & 20,40 \\
Comprimento padrão $(\mathrm{cm})$ & $11,500-17,50$ & $13,300 \pm 2,00$ & 1,20 \\
Eritrócitos $\left(10^{6} / \mu \mathrm{l}\right)$ & $0,950-2,29$ & $1,708 \pm 0,42$ & 0,25 \\
Hemoglobina $(\mathrm{g} \%)$ & $4,900-12,10$ & $6,600 \pm 2,00$ & 1,20 \\
Hematócrito $(\%)$ & $24,000-45,00$ & $32,500 \pm 7,80$ & 4,60 \\
VCM(\%) & $130,000-355,50$ & $209,800 \pm 69,50$ & 41,10 \\
HCM(pg) & $27,200-55,00$ & $40,200 \pm 5,60$ & 5,60 \\
CHCM $(\%)$ & $11,300-40,30$ & $21,400 \pm 8,40$ & 5,00 \\
\hline
\end{tabular}

O estudo do coeficiente da correlação linear de Pearson mostrou correlação altamente positiva $(\alpha=0,01)$ entre peso total e comprimento padrão, em $P$. mesopotamicus, como o esperado (Tab. II). Os eritrócitos evidenciaram correlação positiva $(\alpha=0,05)$ com o percentual de hemoglobina, entretanto, correlação negativa $(\alpha=0,05)$ com o VCM. O hematócrito foi negativamente correlacionado $(\alpha=0,05)$ com o CHCM, enquanto a hemoglobina foi positivamente correlacionada $(\alpha=0,01)$ com o CHCM. Adversamente, o VCM mostrou correlação altamente negativa $(\alpha=0,01)$ com o CHCM. Entretanto, não foi observado correlação entre os dados biométricos e os parâmetros hematológicos da série vermelha (Tab. II).

Tabela II. Coeficientes da correlação linear de Pearson entre os parâmetros hematológicos eritrocitários e os dados biométricos em $P$. mesopotamicus, mantido em policutivo intensivo.

\begin{tabular}{lcccccccc}
\hline Paråmetros & Peso & Comprimento & Eritróitos & Hemoglobina & Hematócrito & VCM & HCM & CHCM \\
\hline Peso & 1,000 & $0,970^{* *}$ & 0,337 & 0,054 & $-0,240$ & $-0,368$ & $-0,277$ & 0,153 \\
Comprimento & & 1,000 & 0,211 & 0,045 & $-0,313$ & $-0,314$ & $-0,164$ & 0,173 \\
Eritrócitos & & & 1,000 & $0,656^{*}$ & 0,336 & $-0,600$ & $-0,364$ & 0,349 \\
Hemoglobina & & & & 1,000 & $-0,085$ & $-0,560$ & 0,447 & $0,835^{* *}$ \\
Hematócrito & & & & & 1,000 & 0,490 & $-0,489$ & $-0,600$ \\
VCM & & & & & 1,000 & 0,044 & $-0,708^{* *}$ \\
HCM & & & & & & 1,000 & 0,594 \\
CHCM & & & & & & & 1,000 \\
\hline
\end{tabular}

$\left(^{\star}\right) \alpha=0,05 ;\left(^{\star *}\right) \alpha=0,01$.

\section{Caracterização morfológica das células sangüíneas de defesa orgânica}

As células sangüíneas de defesa orgânica de $P$. mesopotamicus foram identificadas e caracterizadas: trombócitos, neutrófilos, monócitos, linfócitos e células granulocíticas especiais (CGE).

Os trombócitos são células fusiformes e menores que os eritrócitos, seu citoplasma é acidófilo e sem granulações, com núcleo também fusiforme (Fig. 1A). 
Os neutrófilos são células predominantemente arredondadas, com citoplasma geralmente abundante, ocupando boa parte da célula. O núcleo é excêntrico, raramente central, na maioria das vezes esférico ou lobulado (Fig. 1A). Os monócitos, são células grandes, de formato esférico, ocasionalmente arredondado ou com certo grau de polimorfismo, com citoplasma intensamente basofílico e na maioria das vezes com prolongamentos citoplasmáticos e vacuolização. Seu núcleo é freqüentemente excêntrico, geralmente alongado, alguns vezes podem ser esféricos (Fig. 1A). Os linfócitos são predominantemente arredondados e de tamanho variado, com citoplasma sem granulações visíveis e basofílico. O núcleo possui forma arredondada, levemente riniforme, com cromatina densa, sendo sua relação com o citoplasma bastante elevada (Fig. 1B). As células granulocíticas especiais são arredondadas, com citoplasma abundante e rico em granulações claras, transparentes e esféricas, que espalham-se homogeneamente. Seu núcleo é pequeno e excêntrico, podendo ser ligeiramente alongado ou arredondado, com uma cromatina grosseira e sem nucléolo (Fig. 1C).

Os valores médio da distribuição freqüencial de células sangüíneas de defesa orgânica em $P$. mesopotamicus estão relacionados na tabela III. Observa-se que trombócitos foram as células mais freqüentes, seguido por linfócitos, neutrófilos, monócitos e células granulocíticas especiais (C.G.E.).

Tabela III. Amplitude de variação (Ax), média \pm desvio padrão (SD) e intervalo de confiança (IC) da distribuição freqüencial das células sangüíneas de defesa em $P$. mesopotamicus, mantido em policultivo intensivo. (C.G.E.) Células granulocíticas especiais.

\begin{tabular}{lrrr}
\hline \multicolumn{1}{c}{ Parâmetros (\%) } & \multicolumn{1}{c}{ Ax } & Média \pm SD & IC \\
\hline Trombócitos & $48,0-79,0$ & $61,4 \pm 10,0$ & 7,5 \\
Linfócitos & $6,0-46,0$ & $21,7 \pm 12,0$ & 1,8 \\
Neutrófilos & $0,0-40,0$ & $10,4 \pm 11,0$ & 6,7 \\
Monócitos & $0,0-11,0$ & $4,7 \pm 3,0$ & 1,2 \\
C.G.E. & $0,0-6,0$ & $1,8 \pm 2,0$ & 6,0 \\
\hline
\end{tabular}

O coeficiente da correlação linear de Pearson mostrou que as células sangüíneas de defesa não apresentaram correlação com os dados biométricos estudados, assim como entre si (Tab. IV).

Tabela IV. Coeficientes da correlação linear de Pearson entre as células sangüíneas de defesa e os dados biométricos em $P$. mesopotamicus, mantido em policultivo intensivo. (C.G.E.) Células granulociticas especiais.

\begin{tabular}{lccccccc}
\hline Paråmetros & Peso & Comprimento & Trombócitos & Linfócitos & Neutrófilos & Monócitos & C.G.E \\
\hline Peso & 1,000 & $0,970^{*}$ & 0,051 & $-0,025$ & $-0,080$ & 0,214 & 0,183 \\
Comprimento & & 1,000 & $-0,004$ & $-0,021$ & $-0,037$ & 0,226 & 0,139 \\
Trombocitos & & & 1,000 & $-0,505$ & $-0,554$ & 0,316 & 0,537 \\
Linfócitos & & & & 1,000 & $-0,427$ & $-0,585$ & $-0,505$ \\
Neutrófilos & & & & & 1,000 & 0,148 & $-0,092$ \\
Monocitos & & & & & & 1,000 & 0,213 \\
C.G.E & & & & & & 1,000 \\
\hline
\end{tabular}

$\left(^{\star}\right) \alpha=0,01$. 

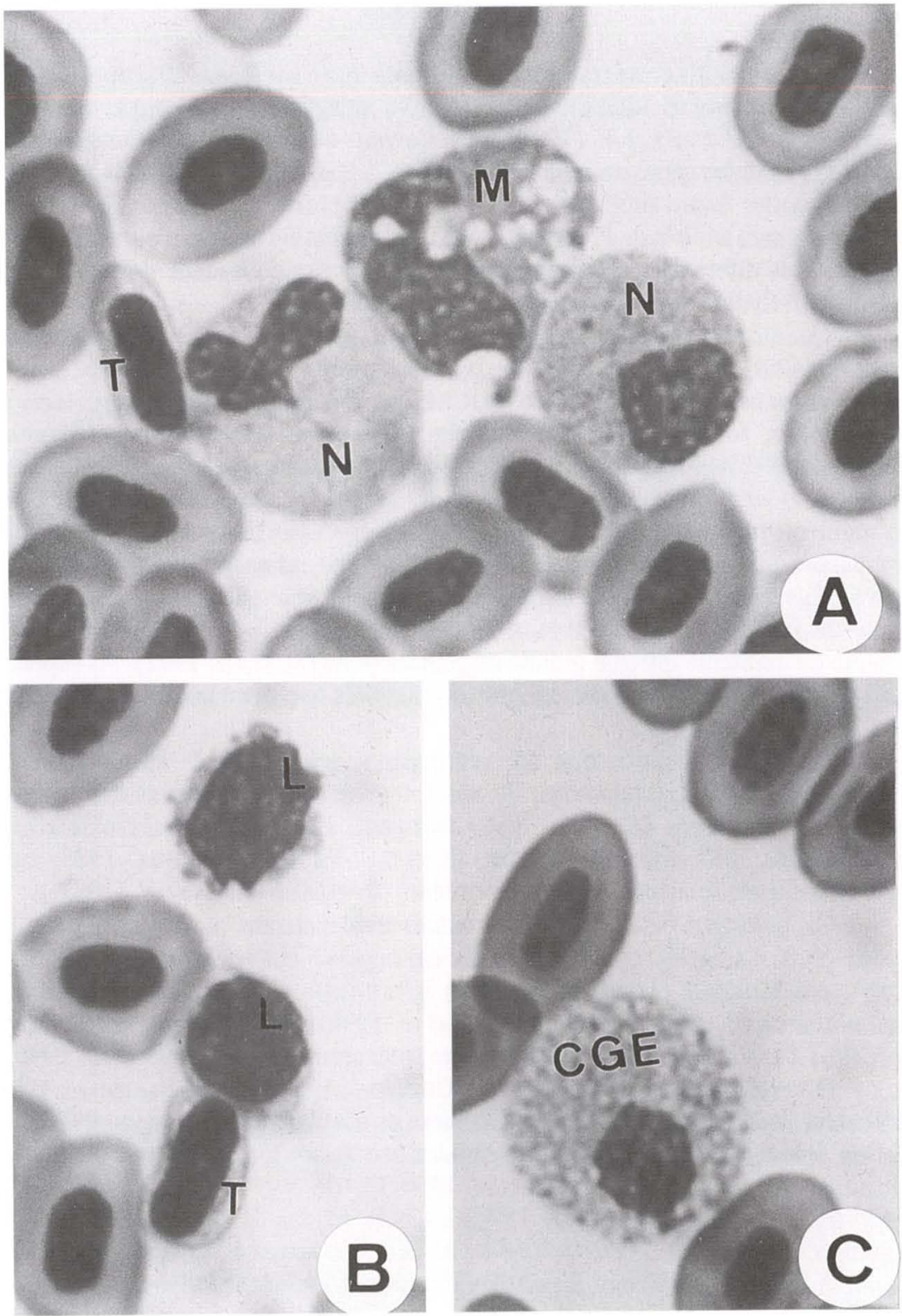

Fig. 1. Células sangüineas de defesa orgânica em Piaractus mesopotamicus. (N) Neutrófilo, (M) monócito, (T) trombócito, (L) linfócito, (CGE) célula granulocitica especial. Coloração: Rosenfeld. 


\section{DISCUSSÃO}

Os resultados deste trabalho demonstram que a contagem total de eritrócitos em $P$. mesopotamicus, mantido em policultivo intensivo, foi maior que os valores descritos por MARTINS et al. (1995), para alevinos da mesma espécie mantidos em aquários e tratados com vitamina C. Entretanto, a taxa de hemoglobina e percentual de hematócrito foram similares. ZUIM et al. (1988) relatam percentual de hematócrito maior para adultos de $P$. mesopotamicus mantidos em cativeiro ou em ambiente natural. Tais diferenças, possivelmente podem ser devido à idade dos animais, já que neste trabalho empregou-se espécimes juvenis.

Quanto aos índices hematimétricos absolutos obtidos para P. mesopotamicus deste trabalho, eles são superiores aos valores descritos por MARTINS et al. (1995) para essa mesma espécie, exceto o CHCM, que foi similar. Valores superiores ao aqui obtidos, foram descritos no também Characidae Colossoma macropomum Cuvier, 1818 (TAVARES-DiAs et al. 1998) e no Loricariidae do gênero Hypostomus (SATAKE et al. 1986; TORRES-FILHO et al. 1986). Entretanto, o cascudo possui eritrócitos com maior diâmetro $(921,29 \mu \mathrm{m})$ (FAVARETO et al. 1978) quando comparado ao $P$. mesopotamicus $(96,90 \mu \mathrm{m})$, como relatado por MARTINS et al. (1995). Contudo, o cascudo é um peixe que possui respiração aérea de emergência (CARTER 1935) e vive em ambiente lêntico, enquanto o $P$. mesopotamicus é uma espécie de ambiente lótico e grande migradora (CASTAGNOLLI 1992). As espécies mais ativas apresentam células sangüíneas menores que as menos ativas (PÉREZ et al. 1984).

As células identificadas no esfregaços sangüíneo de $P$. mesopotamicus, mantido em policultivo intensivo, são morfologicamente similares as descritas em Pimelodus maculatus Lacépedé, 1803 (RIBEIRO 1991), em Prochilodus scrofa Steindachner, 1881 (Prochilodontidae) (RANZANI-PAIVA \& GodinHo 1983) e em Cyprinus carpio Linnaeus, 1758 (Cyprinidae) (RANZANI-PAIVA et al. (1987).

Os esfregaços de $P$. mesopotamicus evidenciaram maior freqüência de trombócitos, à semelhança do que ocorre em Lepomis macrochirus Raf (MURRAY 1984), em Gudusia chapra (Clupeidae) (CHONDAR 1982) e em Sarotherodon melanotheron (Cichlidae) (LEA MASTER et al. 1990). Por outro lado, o número de linfócitos é maior que o de trombócitos em truta arco-íris (HOUSTON et al. 1996).

Diversos autores (MURRAY 1984; CHONDAR 1982; LEA MASTER et al. 1990; HousTON et al. 1996) erroneamente incluem os trombócitos na contagem diferencial de leucócitos. Os trombócitos, embora não sejam leucócitos tem função de defesa em aves e peixes (GRECCHI et al. 1980; ISHIDA et al. 1985; KAJIGAYA et al. 1985; MATUSHIMA \& MARIANO 1996) e são as células de maior prevalência no exsudato inflamatório de Oreochromis niloticus Trewavas 1983 (Cichlidae), com clara evidência de atividade fagocítica (SUZUKI 1986; MATUSHIMA \& MARIANO 1996). Por isso não podem ser considerados responsáveis somente pelo processo de coagulação. Para corrigir tal imperfeição, neste trabalho, foram incluídos em um único bloco denominado de células sangüíneas de defesa orgânica.

Em P. mesopotamicus mantido em policultivo intensivo, o peso total e o comprimento padrão evidenciaram correlação altamente positiva, como o esperado. Porém, esses por sua vez não mostraram correlação com os parâmetros eritrocitários 
e as células sangüíneas de defesa. Contudo, houve correlação entre os parâmetros eritrocitários estudados. Similarmente, estudos em Mugil plantatus Günther, 1880 (RANZANI-PAIVA 1995), em Brycon sp. (Characidae) (RANZANI-PAIVA 1991a) e em C. macropomum (TAVARES-DiAs et al. 1998) também não correlacionam parâmetros hematológicos e dados biométricos. Por outro lado, outros autores puderam correlacionar parâmetros hematológicos com o peso e comprimento (KAVAMOTO et al. 1983; AL-HASSAN et al. 1993).

Os resultados deste estudo sugerem que em $P$. mesopotamicus, mantido em policultivo intensivo, as células sangüíneas de defesa orgânica são morfologicamente similares às descritas para outras espécies. Entretanto, quantitativamente alguns parâmetros sangüíneos aproximam-se ou distanciam-se daqueles descritos na literatura para essa e outras espécies. Isso demonstra a existência de variações na composição sangüínea entre peixes de mesma espécie ou de espécies diferentes. Em teleósteos, os valores hematológicos podem ser influenciados por uma série de fatores, como o sexo, o comprimento, o peso, o estado nutricional, a condição de higidez, o ciclo sazonal e a idade (LARSSON et al. 1976; RANZANI-PAIVA 1991b), assim como o ambiente na qual o animal é mantido (HICKEY 1982; CHAUDHURI et al. 1986; Zuim et al. 1988).

\section{REFERÊNCIAS BIBLIOGRÁFICAS}

ALDRIN, J.F.; J.L. MESSAGER; F. BAUDIN LAURENCIN. 1982.La biochimie clinique en aquaculture. Interet et perspective. CNEXO Actes Colloq.14: 219-326.

Al-Hassan, L.A.J.; H.K. AHMED \& S.A. MAJEeD. 1993. Some haematological parameters in relation to the biology of the fish Acanthopagrus latus. Jour. Environ. Sci. Health 28A (7): 1599-1611.

BANZATO, D.A. \& S.N. KRONKA. 1995. Experimentação agrícola. Jaboticabal, Ed. Funep, $3^{a}$ ed., $247 p$.

CARTER, G.S. 1935. Respiratory adaptation of the fishes of the forest water, with description of the acessory organs of Eletrophorus eletricus (Linn.) and Plecostomus plecostomus (Linn.). Jour. Linn. Soc. Lond. Zool. 39: 219.

CASTAGNOLli, N.1992. Piscicultura de água doce. Jaboticabal, Ed. Funep, 189p.

Chaudhuri, S.H.; T. PANDIT \& S. BANERJEE. 1986. Size and sex related variations of some blood parameters of Sarotherodon mossambica. Environ. Ecol. 4 (1): 61-63.

CHONDAR, S.L. 1982.The haematology of Gudusia chapra (Pisces: Clupeidae). Jour. Inland Fish. Soc. India. 14 (1): 1-10.

CollieR, H.B. 1944. The standardization of blood haemoglobin determinations. Can. Med. Ass. Jour. 50: 550-552.

Favaretto, A.L.V.; R.A. LoPes; S.O. PetenusCi \& P. SaWAya. 1978. Estudo morfométrico de eritrócitos de Plecostomus regani (Pisces, loricariidae). Teleósteo de respiração aquática e aérea, após exposição ao ar atmosférico. Rev. Fac. Farm. Odont., Ribeirão Preto, 15: 59-66.

GoldENFARB, P.B.; F.P. BOWYER; HALL \& E. BRosious. 1971. Reproducibility in the hematology laboratory: the microhematocrit determination. Amer. Jour. Clin. Path. 56: 35-39. 
GRECCHI, R.; A.M. SALIBA \& M. MARIANO. 1980. Morphological changes, surface receptors and phagocytic potencial of fowl mononuclear phagocytes and trombocytes in vivo and in vitro. Jour. Pathol. 130: 23-31.

HiCKEY JR., C.R.. 1982. Comparative hematology of wild and captive cunners. Trans. Amer. Fish. Soc. 111 (2): 242-249.

Houston, A.H.; N. Dobric \& R. KahuranangA. 1996. The nature of hematological response in fish. Fish Physiol. Biochem. 15 (4): 339-347.

IshidA, Y.; M. Sugriama; H. KaJigAYA; M. UmedeA \& M. ISODA. 1985. Inflammatory reactions on Japanese quails muscular tissue caused by sutures. Bull. Nippon Vet. Zoot. Col. 31: 39-46.

Kajigaya, H.; M. Kamekura; N. Tanahara; A. Otha; H. Suzuki; M. SugiyaMA \& M. IsOSA. 1985. Acute and chronic inflammation induced by silk sutures in chicken mesentery. Bull. Nippon Vet. Zoot. Col. 34: 35-8.

Kavamoto, E.T.; M. Tokumaru; R.A.P. Souza E Silva \& B.E.S. Campos. 1983. Algumas variáveis hematológicas do "cascudo" Plecostomus albopunctatus (Regan, 1908). Bol. Inst. Pesca, São Paulo, 10: 101-106.

LARSSON, A.; M.J. JoHANSSON-SJOBECK \& R. FANGE. 1976. Comparative study of some haematological and biochemical blood parameters in fishes from Shagerrak. Jour. Fish Biol. 9: 425-440.

Lea Master, B.R.; J.A. BRocK; R.S. FujioKa \& R.M. NAKAMURA. 1990. Hematologic and blood chemistry values for Sarotherodon melanotheron and a red hybrid tilapia in freshwater and seawater. Comp. Biochem. Physiol. 97A (4): 525-529

Martins, M.L.; N. CAstagnolli; S.M.F. Zuim \& E.C. URBinati. 1995. Influência de diferentes níveis de vitamina $C$ na ração sobre parâmetros hematológicos de alevinos de Piaractus mesopotamicus Holmberg (Osteichthyes, Characidae). Revta bras. Zool. 12 (3): 609-618.

Matushima, E.R. \& M. MARIANO. 1996. Kinetics of the inflammatory reaction induced by carrageenin in the swinbladder of Oreochromis niloticus (Nile tilapia). Braz. Jour. Vet. Anim. Sci. 33 (1): 5-10.

MURRAY, S.A. 1984. Hematological study of the bluegill, Lepomis macrochirus Raf. Comp. Biochem. Physiol. 78A (4): 787-791.

PÉREZ, J.E.; A. BOABA \& G. OJEDA. 1984. Blood parameters in fishes. III. Hemoglobin concentration, hematocrit, and th number of red blood cells in some freshwater fishes of eastern Venezuela. Bol. Inst. Oceanogr. Venezulea. Univ. Oriente 23: 43-47.

RANZANi-PAIVA, M.J.T. 1991a. Características sangüíneas da pirapitinga do sul, Brycon sp, sob condições experimentais de criação intensiva. Braz. Jour. Vet. Res. Anim. Sci., São Paulo, 28 (2): 141-153.

1991b. Hematologia de peixes; p.65-70. In: H.S.L. SANTOS (Ed.). Histologia de Peixes. São Paulo, FCAV-UNESP, 83p.

- 1995. Características hematológicas de tainha Mugil platanus Günther, 1880 (Osteichthyes, Mugilidae) da região estutário-laguna de Cananéia-SP. (Lat. 2500'S - Long. 475' W). Bol. Inst. Pesca, São Paulo, 22 (1): 1-22.

Ranzani-Paiva, M.J.; C.M. Ishikawa; M.C. Portella \& R.J. CeliberTo. 1987. Hematologia da carpa comum Cyprinus carpio, infestada por Argulus sp. e após 
um tratamento com fosfato de 0,0-dimetil-oxi-2,2,2,-tricloroetilo (Neguvon). Bol. Inst. Pesca, São Paulo, 14: 83-92.

RanZANi-Paiva, M.J.T. \& H.M. GodinHo. 1983. Sobre células sangüíneas e contagem diferencial de leucócitos e eritroblastos em curimbatá, Prochilodus scrofa Steindacher, 1881 (Osteichthyes, Cypriniformes, Prochilodontidae). Rev. Brasil. Biol. 43 (4): 331-338.

Ribeiro, W.R. 1991. Leucócitos de peixes, p.61-64. In: H.S.L. SANTOS (Ed.). Histologia de Peixes. São Paulo, FCAV-UNESP, 83p.

RosenfelD, G. 1947. Corânte pancrômico para hematologia e citologia clínica. Nova combinação dos componentes do May-Grunwald e do Giensa num só corante de emprego rápido. Mem. Inst. Butantan 20: 329-334.

Satake, T.; A. NutTi-Sobrinho; O.V. Paula-Lopes; R.A. Lopes \& H.S.L. SANTOS. 1986. Haematological study brazilian fish. III. Blood parameters in armored catfishe Hypostomus paulinus Ihering 1905 (Pisces, Loricariidae). Ars Veterinaria 2 (2): 179-183.

SUZUKI, K. 1986. Morphological and phagocytic characteristics of peritoneal exudate cells in tilapia, Oreochromis niloticus (Trewavas), and carp, Cyprinus carpio L. Jour. Fish Biol. 29 (3): 349-364.

TAVARES-Dias, M.; E.F.S. SANDRIM \& A. SANDRIM. 1998 Características Hematológicas do tambaqui (Colossoma Macropomum) Cuvier, 1818 (Osteichthyes: Characidae) em sistema de monocultivo intensivo. I. Série eritrocitária. Rev. Brasil. Biol. 58 (2): 197-202.

Torres-Filho, I.P.; E.G. Moura; C.C.A. Nascimento; J. Contaifer; C.F. Ramos; M.A. Pimenta \& E.B. Torres. 1986. Parâmetros hematológicos do cascudo (Hypostomus punctatus). Ciênc. Cult. 38: 825-832.

WINTROBE, M.M. 1934. Variations on the size and haemoglobin content of erythrocytes in the blood various vertebrates. Foglia Haematol. 51: 32-49.

ZUIM, S.M.F.; A.A.M. Rosa \& N. CASTAGNOLLI. 1988.Influence of sex and environment on metabolic parameters of pacu Piaractus mesopotamicus (Holmberg, 1887) during final maturation stage. Bull. Canad. Aquacult. Assoc. Proc., Vancouver, 88 (4): 55-56.

Recebido em 10.X.1997; aceito em 29.III.1999. 